Cockcroft 07-36

\title{
RF CAVITY DEVELOPMENT FOR FFAG APPLICATION ON ERLP AT DARESBURY
}

E. Wooldridge, C, Beard, P. McIntosh, B. Todd, B. Fell, ASTeC, STFC Daresbury

Laboratory, Warrington, UK

B. Spencer and R. Jones, Cockcroft Institute \& University of Manchester, Manchester, UK 


\title{
RF CAVITY DEVELOPMENT FOR FFAG APPLICATION ON ERLP AT DARESBURY
}

\author{
E. Wooldridge, C, Beard, P. McIntosh, B. Todd, B. Fell, ASTeC, STFC Daresbury Laboratory, \\ Warrington, UK \\ B. Spenser and R. Jones, Cockcroft Institute \& University of Manchester, Manchester, UK
}

\section{Abstract}

Funding for a non-scaling, Fixed Field Alternating Gradient (FFAG) facility has been approved for installation on the Energy Recovery Linac Prototype (ERLP) at Daresbury. The RF system specification for this project requires the development of a high efficiency, $1.3 \mathrm{GHz}$, normal conducting accelerating structure, capable of delivering the required accelerating voltage, whilst adhering to stringent space limitations imposed by the extremely compact nature of the FFAG ring. We have optimised a cavity design, providing the necessary acceleration and minimising the RF power requirements to match with commercially available power sources.

\section{EMMA}

EMMA (Electron Machine for Many Applications) is a proof-of-principle non-scaling FFAG designed to accelerate electrons from ERLP[1]. The electrons are extracted from ERLP after the first pass through the main linac and injected into EMMA at an energy of $10 \mathrm{MeV}$. A schematic of EMMA is shown in Figure 1 below, this image also shows the first arc of ERLP and the extraction line from ERLP to EMMA.

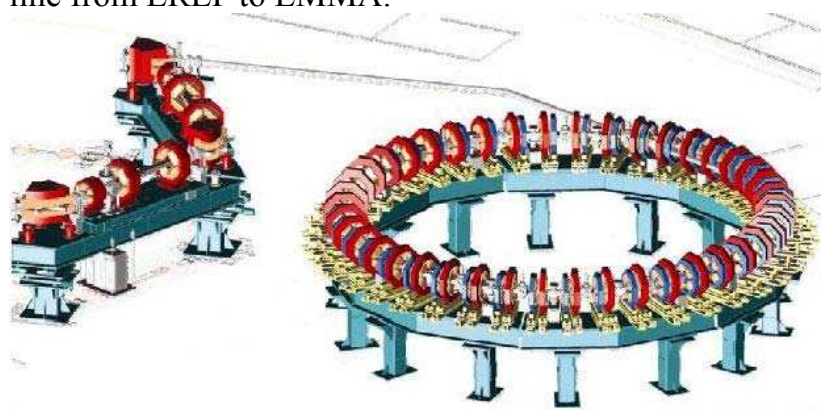

Figure 1: Schematic of EMMA.

EMMA consists of 21 cells, each containing one vacuum pump, two focusing quadrupoles, two defocusing quadrupoles and one RF cavity. These cells are contained within a circumference of $18 \mathrm{~m}$, which the electrons will traverse 10 times accelerating the electrons by approximately $10 \mathrm{MeV}$. Two cavities have been removed from the ring to allow for injection and extraction of the beam, breaking the symmetry contained within. An EMMA cell can be seen in Figure 2 below.

The RF system will operate at $1.3 \mathrm{GHz}$ to complement the ERLP RF system and for the compact size of the accelerating cavities. The requirements of the RF system are fairly stringent due to the size and scope of the machine. Due to the changing beam orbit the cavity must have a large aperture and due to the large number of components within the machine the cavity has a longitudinal space constraint of $105 \mathrm{~mm}$. The nominal cavity voltage is $120 \mathrm{kV}$, giving a total ring voltage of $2.28 \mathrm{MV}$ for the ring, although this may be upgraded in the future. Possible upgrades include increasing the cavity voltage to $180 \mathrm{kV}$ To obtain these higher ring voltages in the future the cavity and distribution needs to be optimised so that these upgrades require a minimum amount of modification.

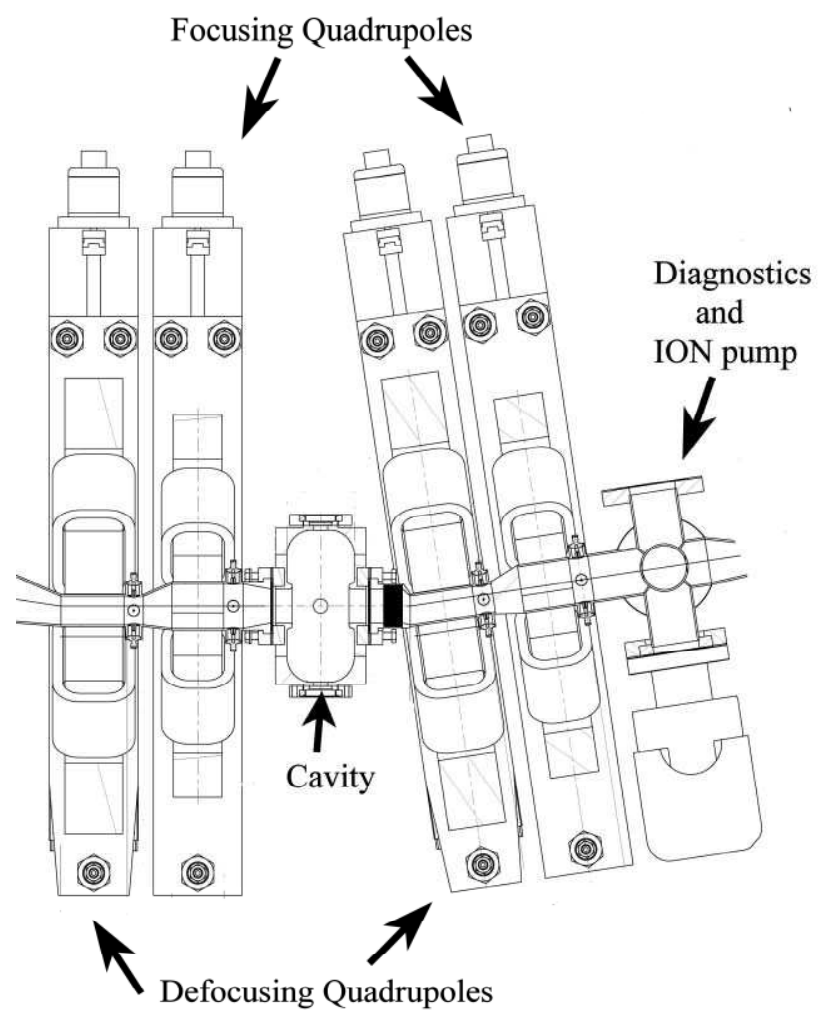

Figure 2: EMMA Cell.

\section{CAVITY OPTIMISATION}

As previously mentioned the cavity must be short, with large beam pipes and possible future upgrades may require the cavity to be operated at up to $180 \mathrm{kV}$. Therefore one of the goals of cavity optimisation is to increase the shunt impedance, $\mathrm{R}_{\mathrm{sh}}$. Enlarging the beam pipes will decrease this figure of merit so the shape must be optimised to counteract this effect. By increasing $R_{s h}$ the power required to operate the cavity will decrease, allowing the voltage to be increased, without necessarily 
needing to increase power architecture. The equation for shunt impedance is given in (1) below.

$$
R_{s h}=\frac{V^{2}}{2 P}
$$

The baseline cavity design for EMMA was the ELBE cavity [2] which was designed as a bunching cavity for the ELBE machine a Forschungszentrum Dresden (FZD). It is a $1.3 \mathrm{GHz}$ cavity designed to be run in $\mathrm{CW}$ (Continuous Wave) mode and as originally designed this cavity has a shunt impedance of $1.4 \mathrm{M} \Omega$ therefore, from equation 1 , to obtain $120 \mathrm{kV}$ per cavity would require 5.1 $\mathrm{kW}$.

Three cavities were investigated for use in EMMA. One was a modified ELBE cavity, another was based on the PEP II cavity geometry [3], and the third was based on a simple torus. The PEP II cavity was originally designed as a $476 \mathrm{MHz}$ cavity and therefore had to be scaled to 1.3 GHz. To modify each of these designs several variables were changed, shown in Figure 3 below; for all these models cavity frequency at was maintained at $1.3 \mathrm{GHz}$.

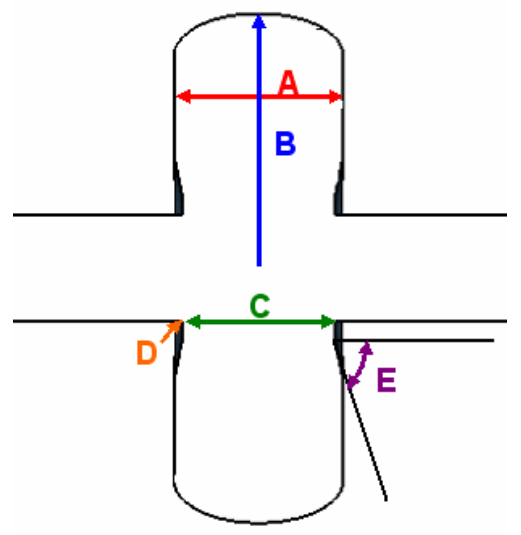

Figure 3: Variables in EMMA cavity optimisation.

Due to size constraints the cavity can only be $105 \mathrm{~mm}$ long from flange to flange, giving an internal accelerating length of $\sim 86 \mathrm{~mm}, \mathrm{C}$, and since the beam orbit changes the beam pipe radius must be large, $20 \mathrm{~mm}$. Increasing the cavity width, $\mathrm{C}$ in Figure 2, was seen to increase the shunt impedance. Increasing the angle of the nose cones, labelled E in Figure 3, caused the shunt impedance to decrease and no significant change was observed when the radius of the nose cones, $\mathrm{D}$, was changed. The changes in the ELBE and PEP II cavities options can be seen in Figures 4 and 5 respectively. These show the initial cavity design on the left hand side and the cavity with improved shunt impedance on the right.

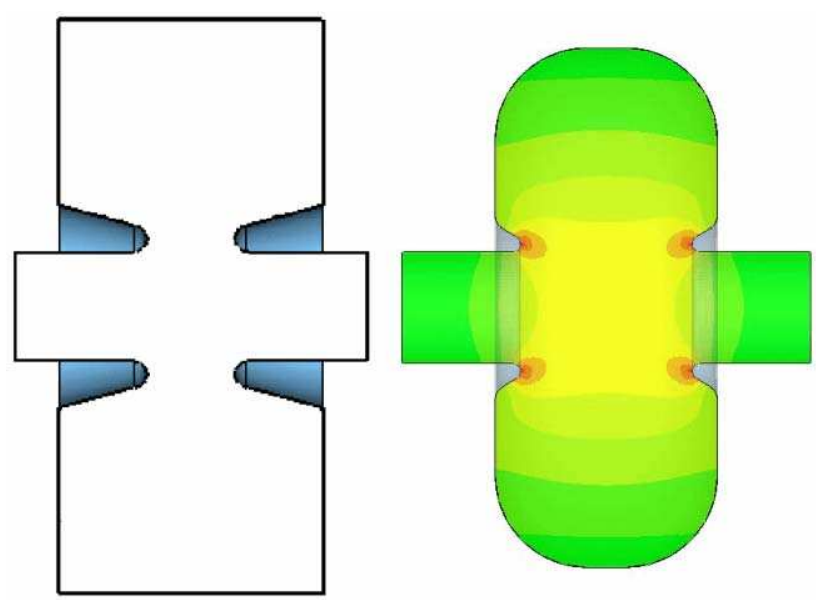

Figure 4: Changes to the ELBE type cavity.
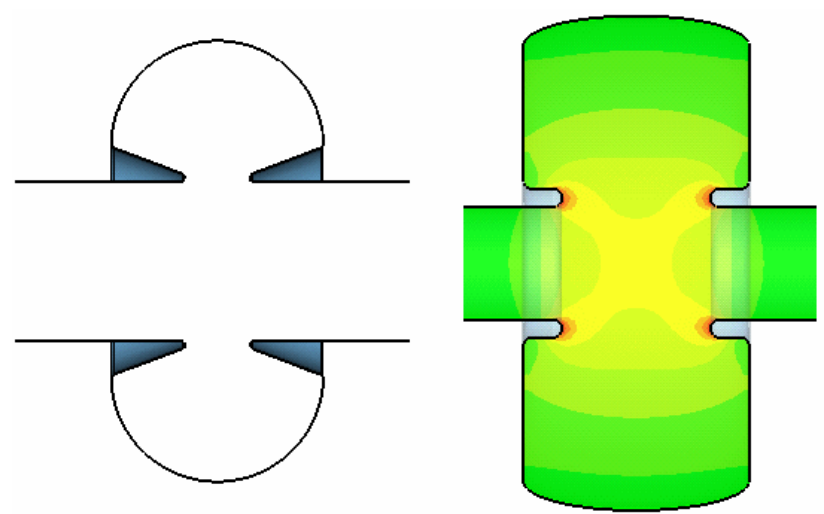

Figure 5: Changes to the PEP II type cavity.

It can be seen in Figures 4 and 5 that the two cavity designs are starting to converge. The equator region of the ELBE type cavity has been rounded off and the PEP II type cavity has minimised the nose cone angle. The next step was to model a cavity made from a torus intersected by the beam pipe. This gave a greatly improved shunt impedance as can be seen from Table 1 below. The final toroid design, values given in the last column of Table 1, has a cavity length of $96 \mathrm{~mm}$ as additional space for the cavities has become available due to a change in the flanges.

Table 1: Cavity Shunt Impedance and Power Requirements

\begin{tabular}{|l|l|l|l|l|l|}
\hline & $\begin{array}{l}\text { ELBE } \\
\text { Cavity }\end{array}$ & $\begin{array}{l}\text { ELBE } \\
\text { Like } \\
\text { Design }\end{array}$ & $\begin{array}{l}\text { PEP II } \\
\text { Like } \\
\text { Design }\end{array}$ & $\begin{array}{l}\text { Toroid } \\
\text { Design }\end{array}$ & $\begin{array}{l}\text { Final } \\
\text { Toroid } \\
\text { Design }\end{array}$ \\
\hline Shunt Impedance $/ \mathrm{M} \Omega$ & 1.4 & 2.52 & 2.4 & 3.41 & 4.3 \\
\hline Practical Shunt Impedance $/ \mathrm{M} \Omega$ & 1.12 & 2.016 & 1.92 & 2.728 & 3.44 \\
\hline Power Requirements @ $120 \mathrm{kV} / \mathrm{kW}$ & 6.43 & 3.57 & 3.75 & 2.64 & 2.09 \\
\hline Power Requirements @ $180 \mathrm{kV} / \mathrm{kW}$ & 14.46 & 8.04 & 8.44 & 5.94 & 4.71 \\
\hline
\end{tabular}


To confirm these results the fields and the shunt impedance was calculated in Microwave Studio (MWS) [4] and HFSS [5]. The field are plotted in Figure 6 below, the MWS plot is on the left, the HFSS plot on the right.

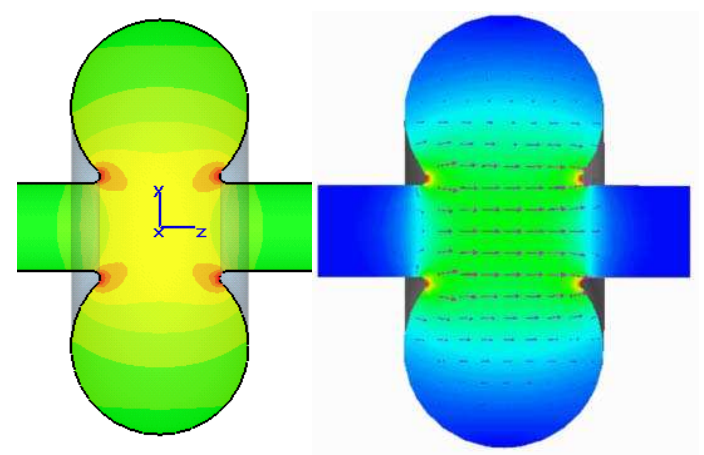

Figure 6: Field Plots for the Toroid Cavity.

The shunt impedance was calculated to be $4.3 \mathrm{M} \Omega$ in MWS and 4.2 M $\Omega$ in HFSS. This is a theoretical impedance, in practice this value would be approximately $20 \%$ lower, around $3.4 \mathrm{M} \Omega$, since the cavity will not be made from a perfect electrical conductor and protrusions into the cavity for the tuner and input coupler will reduce its overall $\mathrm{R}_{\mathrm{sh}}$.

\section{RF DISTRIBUTION}

RF power needs to be distributed from one or more sources to each of the cavities. The torus design with a cavity voltage of $120 \mathrm{kV}$ requires, from (1), $2.09 \mathrm{~kW}$ to be delivered to each cavity. When calculating the power requirements for the ring the losses in transmission lines will need to be included. There are multiple sources available to power these cavities in the form of Inductive Output Tubes (IOTs) or klystrons. At $1.3 \mathrm{GHz}$ existing IOTs can supply up to 20 or $30 \mathrm{~kW}$ of power and klystrons can supply up to $120 \mathrm{~kW}$. These are peak power values; it is common for these supplies to be run at lower than peak power where the effects are linear. This is a drop of 1 to $2 \mathrm{~dB}$ for klystrons and $1 \mathrm{~dB}$ for IOTs.

Assuming $2.09 \mathrm{~kW}$ per cavity, the entire ring will require around $40 \mathrm{~kW}$ of power; this is before losses in the distribution to the cavities which will increase the power requirements to $65 \mathrm{~kW}$. For the requirement of $180 \mathrm{kV}$ per cavity the total RF power requirement becomes $90 \mathrm{~kW}$.

A method of distributing this power from its source to the cavity is also required. There are two methods to achieve this; the first is to cascade the distribution from cavity to cavity, this is the preferred solution if the cavities are to be powered by a klystron. This set up is shown in Figure 6 below.

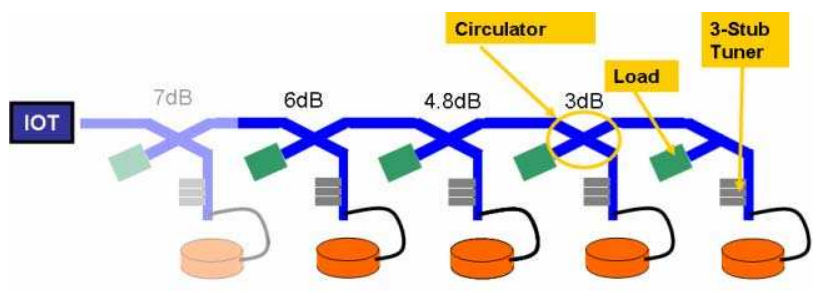

Figure 7: Cascaded distribution.

The second method is to split the power using waveguide tees, this splits the power equally into two separate waveguide feeds. This is repeated until the correct power is reached at each cavity. A diagram of this method is given in Figure 8 below.

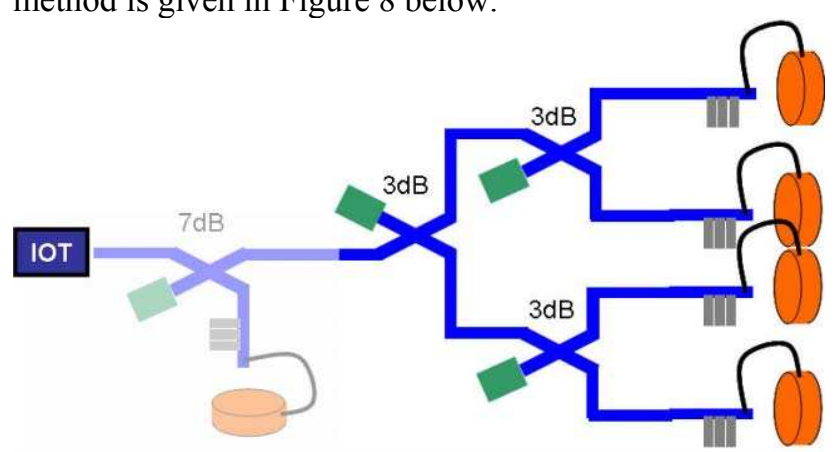

Figure 8: Split distribution.

Although this method can be used for both IOTs and klystrons it is optimised for use with IOTs since splitting power from 1 klystron to 19 cavities in this manner would require a large and cumbersome amount waveguide. Also due to the location of the machine, within the ERLP hall, there are constraints on the available space.

\section{SUMMARY}

EMMA is a concept non-scaling FFAG to be built at Daresbury using the beam from ERLP. The RF requirements for EMMA are challenging, and its cavity has been optimised for high shunt impedance and hence minimising the cavity losses. The cavities will be powered by commercially available klystrons or IOTs. There are two options to distribute the power to the cavity by cascading the power through splitters from cavity to cavity or by dividing the power through splitters until the required number of outputs is achieved. The method used will be dependant on the power source chosen.

\section{REFERENCES}

[1] R. Edgecock, "EMMA - the World's First Non-scaling FFAG", presentation at this conference.

[2] "Stripline Beam Position Monitors For "Elbe", Proceedings of DIPAC 2001, ESRF, Grenoble, France, 2001.

[3] H.D. Schwarz et al., "Design of a High-Power Test Model of the PEP-II RF Cavity", PAC'93, Washington DC, May 93, http://www.jacow.org.

[4] CST Microwave Studio Code, more information at www.cst.de.

[5] Ansoft HFSS code, more information at www.ansoft.com. 